\title{
PULMONARY EMBOLISM STILL A DIAGNOSTIC QUANDARY
}

\author{
Islam $\mathrm{T}^{1}$
}

\begin{abstract}
Objectives: The objective was to review the progress made in the diagnosis of pulmonary embolism over the past decade and the future direction of diagnostic modalities.

Methods: Research studies, review articles and scientific meeting abstracts published were reviewed.

Results: Significant progress has been made in the diagnosis of pulmonary embolism. Strategies have been developed to increase the sensitivity and specificity of the diagnostic tools.

Conclusion: With the advances of the technology and better understanding of patho-physiology, offer the hope of improving the medical management of common and potentially life threatening condition like pulmonary embolism.
\end{abstract}

Key wards: Pulmonary embolism, diagnostic modalities, progress

\section{Introduction}

Pulmonary embolism (PE) is a common but potentially fatal condition and one of the leading causes of sudden death in the western world. Although the true incidence of PE is unknown, it is estimated that over 100,000 cases in France, 65000 cases among hospitalized patients in England and Wales and at least 600,000 cases in USA occur per year. Although the basic patho-physiology of a pulmonary embolism is understood for many years, the morbidity and mortality associated with this disease remains unacceptably high. Alikhan et al carried out a retrospective review of all postmortem reports during the period 1991 to 2000 at King's College Hospital, London and fatal pulmonary embolism was recorded as cause of death in 265 out of 6833 cases (3.9\% of all necropsies; $5.2 \%$ of adult cases) ${ }^{1}$. Mortality in untreated PE is approximately $25-30 \%$, but with adequate treatment, this can be reduced to $2-8 \%{ }^{2-3}$. Over all mortality reported in International Cooperative Pulmonary Embolism Registry (ICOPHER) study was $45 \%$, whereas 3-month mortality rate for all pulmonary embolism was as high as $17.4 \%{ }^{4}$. Patients with stable PE sometimes do well without treatment ${ }^{5}$. Unfortunately, the diagnosis of PE remains a complex and challenging process for the diagnosis of PE arises from nonspecific clinical presentations, coupled with a host of diagnostic options which all possess inherent limitation. clinicians and radiologists alike. Much of the difficulty in

\section{Risk Factors for Venous Thromboembolism}

The majority of patients with pulmonary emboli have an underlying clinical predisposition. Less than $10 \%$ of the patients have no discernible cause for deep venous thrombosis at the time of presentation. Congenital predisposition to thrombosis is considered to be rare but should be considered in the setting of strong family history, recurrent venous thromboembolism (VTE), deep vein thrombosis (DVT) in unusual site and VTE under the age of 40 years. Pregnancy and hormone replacement therapy (HRT) are both associated with three to five fold increased risk of VTE. Chronic obstructive pulmonary disease (COPD) is considered a risk factor for PE and PE is part of the differential diagnosis of an acute exacerbation of COPD. COPD-PE study is currently undergoing to look at the prevalence of $\mathrm{PE}$ in a patient with acute exacerbation of COPD.

Table-I: Primary risk factors of PE

\begin{tabular}{|l|l|}
\hline Antithrombin deficiency & Protein C deficiency \\
Congenital & Protein S deficiency \\
dysfibrinogenemia & Hyperhomocysteinemia \\
Factor V Leiden & Anticardiolipin antibodies \\
Plasminogen deficiency & \\
Dysplasminogenemia & \\
\hline
\end{tabular}

Table-II: Secondary risk factors

\begin{tabular}{|l|l|}
\hline Surgery (Abdominal/pelvic) & Stroke \\
Immobilization & Advanced age \\
Malignancy & Obesity \\
Heart failure & Long bone fracture \\
Smoking & Long distance travel \\
Pregnancy/Puerperium & Oral contraceptives \\
Lupus anticoagulant & Nephrotic syndrome \\
Prosthetic surfaces & Hyperviscosity \\
\hline
\end{tabular}

Table-III: Definition and Classification of PE

Definition: Obstruction to blood flow to one or more
pulmonary arteries. PE can be classified into three
main groups:
Massive: Consists of shock and/or hypotension
(defined as systolic blood pressure $<90 \mathrm{mmHg}$ or a
pressure drop of $\geqslant 40 \mathrm{mmHg}$ for $\geqslant 15 \mathrm{~min}$ ).
Submassive: Haemodynamically stable but there is
right ventricular dilation or hypokinesia.
Nonmassive: No evidence of shock and righ
ventricular involvement.

1. Dr Tasbirul Islam, MD, MRCP(UK), Consultant, Division of Pulmonary, Critical Care Medicine, Marshfield Clinic, Marshfield, WI 54449, USA. 


\section{Most Commonly Observed Signs and Symptoms}

Unfortunately, the clinical diagnosis of pulmonary embolus is unreliable. The clinical spectrum of pulmonary embolism varies from asymptomatic to fatal outcome. Symptoms and signs of pulmonary embolus are nonspecific and don't correlate with severity or anatomic findings. The classical clinical triad of haemoptysis, pleuritic chest pain and dyspnoea occurs in $20 \%$ or less of cases. Of patients who die from massive PE, only $60 \%$ have dyspnoea, $17 \%$ have chest pain, and $3 \%$ have haemoptysis. The most common clinical manifestations were: dyspnoea ( $92 \%$ of cases), tachycardia $(80 \%)$, syncope (44\%), cardiac arrest $(22 \%)$, and shock $(20 \%)$. Thoracic pain was present in only $27 \%$ of patients ${ }^{6}$. Wells et al studied 930 patients with suspected pulmonary embolism and demonstrated that by combining consideration of pretest clinical probability and the SimplyRED d-dimer test, pulmonary embolism can be diagnosed or ruled out safely, with a dramatic reduction in the need for imaging procedures ${ }^{7}$.

Table-IV: Most common signs and symptoms

\begin{tabular}{|l|l|}
\hline Signs & Symptoms \\
\hline Tachycardia (HR $>100 / \mathrm{min})$ & Dyspnoea \\
Tachypnoea (RR $>16 / \mathrm{min})$ & Pleuritic chest pain \\
Accentuated $2^{\text {nd }}$ heart sound & Apprehension, anxiety \\
Cyanosis & Syncope \\
Fever & Haemoptysis (rare) \\
& Diaphoresis (rare) \\
\hline
\end{tabular}

Table-V: Pretest clinical probability ${ }^{8}$

\begin{tabular}{|l|c|}
\hline Clinical signs and symptoms of DVT & +3.0 \\
\hline An alternative diagnosis less likely than PE & +3.0 \\
\hline Pulse rate $>100 /$ min & +1.5 \\
\hline Immobilization/Surgery in the previous 4 weeks & +1.5 \\
\hline Previous PE/DVT & +1.5 \\
\hline Haemoptysis & +1.0 \\
\hline $\begin{array}{l}\text { Malignancy (on treatment, treated in the past } 6 \\
\text { months, or palliative) }\end{array}$ & +1.0 \\
\hline $\begin{array}{l}<2=\text { Low pretest probability } 2 \text { to } 6=\text { Moderate pretest } \\
\text { probability }>6=\text { High pretest probability }\end{array}$ \\
\hline
\end{tabular}

\section{Diagnostic Modalities}

No single imaging study or blood test is $100 \%$ sensitive and specific for pulmonary embolism. A combination of pretest clinical probability assessment, noninvasive pulmonary imaging [ventilation-perfusion(V-P) scanning or spiral computed tomography $(\mathrm{CT})$ ] and d-dimer testing seems prudent before pursuing pulmonary angiography.

Table-VI: Diagnostic modalities

\begin{tabular}{|l|l|}
\hline Non-Imaging & Imaging \\
\hline Risk factors & X-ray chest \\
\hline Symptoms & Lung Scanning \\
\hline Signs & Echocardiography \\
\hline ECG & Leg ultrasound \\
\hline d - dimer & Spiral CT scan \\
\hline & Pulmonary angiography \\
\hline
\end{tabular}

\section{Blood Tests}

Unfortunately, no known blood or serum test can move a patient with a high clinical likelihood of pulmonary thromboembolism into a low likelihood category or vice versa. The white blood cell (WBC) count may be normal or elevated. Arterial blood gas may show low partial pressure of oxygen $\left(\mathrm{PO}_{2}\right)$, low Partial pressure of carbon dioxide $\left(\mathrm{PCO}_{2}\right)$ and increase alveolar to arterial gradient (A-a gradient). In a report by Palla and colleagues ${ }^{9}, 15 \%$ to $20 \%$ of patient with proven PE have normal A-a gradient. Stein et $\mathrm{al}^{10}$ reviewed the data from Prospective Investigation of Pulmonary Embolism Diagnosis (PIOPED) I study and found 30\% patient with no cardiopulmonary disease and $14 \%$ patient with prior cardiopulmonary disease had PE with normal arterial blood gas (ABG). Pulse oximetry is extremely insensitive, is normal in the majority of patients with PE, and should not be used to direct a diagnostic workup. Cardiac troponin I may be a useful marker to suggest right ventricular involvement and may indicate increase mortality ${ }^{11-13}$. Similarly raised Brain natriuretic peptides (BNP) may reflects right ventricular overload and predict in hospital complication and mortality ${ }^{14}$.

\section{Electrocardiogram (ECG)}

The most common ECG abnormalities in the setting of $\mathrm{PE}$ are tachycardia and nonspecific ST-T wave abnormalities. Any other ECG abnormality may appear with equal likelihood, but none are sensitive or specific for PE.

Table-VII : Common ECG findings in PE

$$
\begin{aligned}
& \text { m Tachycardia } \\
& \text { m ST-T changes } \\
& \text { m Tall peaked P wave in lead II } \\
& \text { m Right axis deviation } \\
& \text { m Right bundle branch block } \\
& m \mathrm{~S}_{1} \mathrm{Q}_{3} \mathrm{~T}_{3} \\
& \mathrm{~m} \text { Atrial fibrillation }
\end{aligned}
$$

Unfortunately, only $20 \%$ of patients with proven PE have any of these classic ECG abnormalities. The most common electrocardiographic abnormalities were nonspecific $\mathrm{T}$ wave changes which occurred in $42 \%$ of patients and nonspecific abnormalities (elevation or depression) of the RST segment which occurred in $41 \%$ of patients ${ }^{15}$. The anterior subepicardial ischemic pattern is the most frequent ECG sign of massive PE and early reversibility indicates a good outcome ${ }^{16}$. Reversible prolonged Q-T interval may be present in a patient with pulmonary embolism ${ }^{17}$. In massive embolism, the electrocardiogram was normal in $6 \%$ (3 of 50) of patients. With submassive embolism, $23 \%$ of patients $(9$ of 40) had a normal electrocardiogram ${ }^{15}$. Casazza et al ${ }^{6}$ studied 160 cases of confirmed pulmonary embolism and found a right bundle branch block was present in $47 \%$ of cases, T-wave inversion in $32 \%$ of cases and was not 
related to the severity and outcome of pulmonary embolism. New onset right bundle branch block (RBBB) may indicate massive obstruction of the main trunk ${ }^{18}$. Right sided ECG may be helpful in identifying RBBB in presence of normal 12 lead $\mathrm{EKG}^{19}$. If ECG abnormalities are present, they may be suggestive of $\mathrm{PE}$, but the absence of ECG abnormalities has no significant predictive value.

\section{d-dimer}

Plasma d-dimer, a degradation product of cross-linked fibrin can be measured by three method - ELISA, Latex and Whole agglutination (SimplyRED) tests. When assayed by a quantitative ELISA method has been shown highly sensitive ( more than 95\%) in VTE at a cutoff value of $500 \mathrm{ng} / \mathrm{ml}$ but specificity remains low (55\%) as fibrin is produced in a wide variety of conditions, such as cancer, inflammation, infection, necrosis. Hence, a ddimer level above $500 \mathrm{ng} / \mathrm{ml}$ has a poor positive predictive value for $\mathrm{PE}$. In a prospective study involving 96 patient over 70 years old without history of metastatic cancer or recent surgery, trauma, infection, stroke, myocardial infarction, deep vein thrombosis (DVT) or PE found that ELISA d-dimer is as sensitive as in adult population but due to the comorbid conditions, only a few patients presented with d-dimer values less than 500 $\mathrm{ng} / \mathrm{ml}^{20}$. A normal d-dimer level by ELISA assay may safely exclude PE but traditional latex and whole agglutination tests should not be used to rule out PE. A study comparing d-dimer with tissue plasminogen activator (tPA), using a cutoff of $8.5 \mathrm{ng} / \mathrm{mL}$, showed that the sensitivity and negative predictive value of d-dimer were low compared with $\mathrm{tPA}^{21}$. Kucher et $\mathrm{al}^{22}$ tested the hypothesis that ratio of d-dimer/Fibrinogen (D/F) may be more specific than d-dimer alone. Their study showed that the true positive rate was almost twice as high using $\mathrm{D} / \mathrm{F}$ ratio $>1.04 \times 103$ (49 of 85 patients; 57.6\%) compared with d-dimer $>7000$ micro g/L ( 25 of 85 patients; 29.4\%). In summery, d-dimer is an important diagnostic tool when index of suspicion for PE is low and in the setting of high clinical probability, physician should order a more specific test rather that wasting time on d-dimer.

\section{Chest Radiograph}

The value of obtaining preliminary chest X-ray (CXR) in the workup of $\mathrm{PE}$ is in ruling out conditions that might be confused with PE and identifying coexisting cardiopulmonary processes that might interfere with the interpretation of $\mathrm{V} / \mathrm{Q}$ scan. Following are the findings may present in CXR of suspecting PE:

$\mathrm{m}$ Decreased pulmonary vasculature with prominent proximal pulmonary vessels -Westermark Sign. $\mathrm{m}$ Elevated hemidiaphragm.

$\mathrm{m}$ Pleural effusion ( $80 \%$ exudative)

$\mathrm{m}$ Wedge shaped infiltrate (Hampton's hump)-Rare, late finding.

m Often normal.
In a recent review of patients with pulmonary embolus and no pre-existing cardiac or pulmonary disease, only $16 \%$ of the chest $x$-rays were normal ${ }^{23}$. Atelectasis and/or pulmonary parenchymal abnormalities were most common findings $(68 \%)$ and only $6 \%$ had the Westermark sign ${ }^{23}$. Elliot et al reviewed the International Cooperative Pulmonary Embolism Registry (ICOPER) database and found cardiomegaly was reported most common x-ray findings ${ }^{24}$. In the Prospective Investigation of Pulmonary Embolism Diagnosis (PIOPED) study, pleural based wedged shaped infiltrate, oligemia and abrupt cut off of hilar vessels appeared to be closely associated with PE and were present in $15 \%-45 \%$ of patients ${ }^{25}$.

\section{Echocardiography}

Right ventricular failure is the ultimate cause of death in patients who succumb to acute PE. Presently ,echocardiography and doppler analysis of right heart dimensions and RV function permits neither definitive confirmation nor exclusion of suspected PE. However, hemodynamically important $\mathrm{PE}$ is unlikely in a patient with a normal echocardiogram.

Table-VIII: Echocardiographic features of PE

$$
\begin{aligned}
& \text { m Right ventricular hypokinesia } \\
& \mathrm{m} \text { Right ventricular dilation } \\
& \mathrm{m} \text { An increased RV/LV ratio caused by } \\
& \text { interventricular bulging into the LV } \\
& \mathrm{m} \text { Dilated proximal pulmonary arteries } \\
& \mathrm{m} \text { Tricuspid regurgitant jet velocity }>3.7 \mathrm{~m} / \mathrm{s} \\
& \mathrm{m} \text { IVC is usually dilated and doesn't collapse } \\
& \text { on inspiration }
\end{aligned}
$$

This finding has been shown to correlate not only with larger emboli but also recurrence of PE. Those who have above changes on echocardiography with or without features of shock could benefit from thrombolytic therapy. Echo features such as right ventricular (RV) free wall thickness $>5 \mathrm{~mm}$, tricuspid reugitant jet velocity $>3.7 \mathrm{~m} / \mathrm{s}$ and dilated RV with normal interventricular septal motion may also helpful to differentiate acute from subacute massive $\mathrm{PE}^{26}$. Relative sparing of the right ventricular apex , known as McConnell sign sometimes useful to differentiate between right ventricular dysfunction due to PE or due to other condition ${ }^{27}$. Pruszczyk et al studied 49 patients with suspected massive pulmonary embolism and found that sensitivity of Trans esophageal echo (TEE) was $80 \%$ for detecting PE of any size and specificity was $100 \%{ }^{28}$. In one study, $92 \%$ of patient with $30 \%$ or more impaired lung perfusion develop right ventricular hypokinesia ${ }^{29}$. Mortality is increased in patient with right ventricular involvement which sustained up to three months after diagnosis of PE. Right ventricular dysfunction may provide a powerful predictor of inhospital death, with a 6-fold increase in relative risk compared with normal right ventricular function ${ }^{30}$. Similar results were obtained in ICOPER ${ }^{4}$. Overall, $5 \%$ developed 
chronic pulmonary hypertension. Some authors suggest follow up TEE has been utilized to document emboli in the main or right pulmonary artery. At present, the role of transthoracic Echo for the diagnosing of acute PE remains undefined and can't be considered a first line diagnostic tool for investigation of PE. But certainly will influence the treatment and outcome/prognosis.

\section{Venous Doppler Ultrasound}

Since PE is a complication of deep vein thrombosis (DVT). Most of the data showed that $90 \%$ of PEs originates from lower extremity DVT, doppler ultrasonography often has an instrumental role in the workup of suspected PE. Duplex scan only reliably identify DVT in the thigh and groin (sensitivity and specificity in symptomatic patient 95 and $98 \%$ respectively) and are poor in visualizing pelvis and calf veins. One study ${ }^{31}$ evaluates the utility of venous duplex imaging as the initial test in a diagnostic algorithm for patients with suspected PE. With unilateral leg symptoms, DVT was found in $40 \%$ and without unilateral leg symptoms, results of venous duplex imaging (VDI) were abnormal in only $5 \%{ }^{31}$. DVT was found in $25 \%$ of patients with high-probability V-P scans and in 25\% with non-highprobability scans and conclusion was VDI appears to be a reasonable initial screening test in patients with clinically suspected PE and unilateral leg symptoms ${ }^{32}$. Retrospective study involving 136 patients, comparing VDI with computed tomography venography (CTV) found CTV is less sensitive and more costly than $\mathrm{VDI}^{30}$. Overall, lower extremity sonography only detects DVT in approximately $50 \%$ of patients with documented PE.

Another limitation of sonography is to differentiate between acute vs chronic DVT. Findings suggestive of chronicities includes collateral vein, small caliber vein, irregular vessel wall, increased clot echogenicities ${ }^{33,34}$. Serial leg testing may replace angiography in patients with non-diagnostic lung scan findings. However, its practical use seems limited. In one study with non-high probability V-P scan, they found that sensitivity of lower extremities colour flow venous duplex examination.

Table-IX : Factors that reduce the positive predictive value of venous ultrasonogram

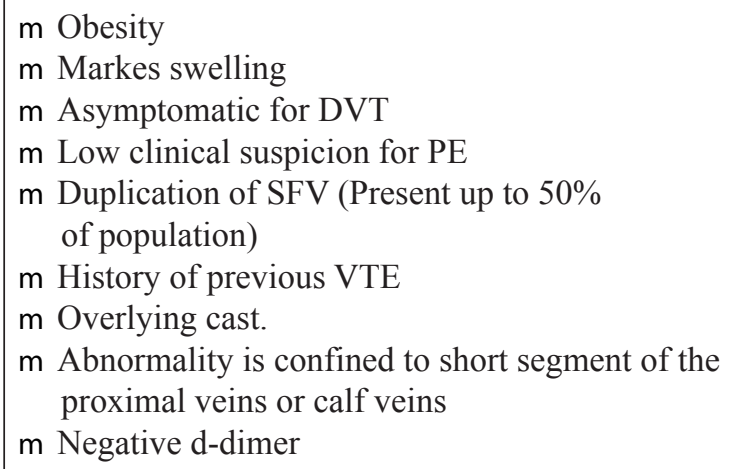

alone is $44 \%$ and increase to $62 \%$ when combined with V-P $\operatorname{scan}^{35}$. Similar results showed in a study by Daniel et al even in a best-case scenario ${ }^{36}$.

\section{Ventilation-Perfusion (V-P) Scan}

V-P scan have long been a mainstay in the workup of patients with suspected PE. The reason for this are two fold: it is a non-invasive diagnostic technique and it has been evaluated in extensive clinical trials. PIOPED I ${ }^{37}$ study has established the value of V-P lung scan. The prevalence of $\mathrm{PE}$ with normal perfusion scan is so small $(<0.2 \%)$ that a normal scan virtually excludes the diagnosis. Similarly, the likelihood of PE with low probability results is estimated to be $4 \%$. Whereas a high probability V-P scan is considered diagnostic in the setting of appropriate clinical scenario. But unfortunately large fraction of $\mathrm{V} / \mathrm{Q}$ scan (60 to $70 \%$ ) resides in a grey area that is characterized by either indeterminate or intermediate probability interpretation. Approximately $25 \%$ of patients with suspected PE will have the diagnosis refuted by a normal perfusion scan and anticoagulants may be safely withheld. Around $25 \%$ of patients with suspected PE will have a high probability lung scan and anticoagulant may be instituted. The remaining patients will require further diagnostic tests as those patient have a $20 \%$ to $40 \%$ prevalence of angiographically proven PE. The $67 \%$ specificity and 61 to $70 \%$ interobserver agreement rates illustrate the diagnostic limitations of these scans ${ }^{38}$. Gotthardt et $\mathrm{al}^{39}$ found, out of the 129 patients receiving a scintigraphic follow-up, only 29 (22.5\%) had a follow-up over more than 1 year, 19 of those had persisting perfusion defects. It is concluded that an inadequate scintigraphic followup of patients with pulmonary embolism which may lead to unnecessary anticoagulant treatment if persisting perfusion defects are misinterpreted as fresh pulmonary embolism $^{39}$. Ventilation-perfusion scanning provides a definitive diagnosis in fewer than $40 \%$ of $\operatorname{cases}^{37,40}$. These limitations may explain why clinicians often do not pursue definitive objective tests for suspected pulmonary embolism ${ }^{41-43}$. Abnormal X-ray chest (CXR) or chronic lung disease is another diagnostic challege for V-P scan. Scot et al found that computer-based V-P scan (Using ANN) analysis performs comparably to clinical interpretation for patients with abnormal chest radiographs ${ }^{44}$. Stein et al conducted study in a population with or without cardiac or pulmonary disease and results of V-P. They found the utility of V-P scan was not impaired in presence of pre-existing cardiac or pulmonary disease except fewer patien without history of cardiopulmonary disease had intermediate V-P $\operatorname{scan}^{45}$. Retrospectively analyzed up to one year of follow-up in 90 patients who were clinically suspected of having PE, but in whom V-P scintigraphy implied a low probability of PE. None of the 90 patients demonstrated clinical evidence of PE subsequent to the V-P $\operatorname{scan}^{46}$. In PISAPED, they prospectively evaluated 890 consecutive 
patients with suspected pulmonary embolism. Of 890 scan, 670 were classified as abnormal. A definitive diagnosis was established in $563(84 \%)$ patients with abnormal scans and the conclusion was accurate diagnosis of pulmonary embolism is possible by perfusion scanning alone, without ventilation imaging ${ }^{47}$. In a report by Reinartz and his colleagues ${ }^{48}$, V-P lung scintigraphy using SPECT and ultrafine aerosols technique and helica CT are comparable diagnostics accuracy.

\section{CT Scan}

Until the early 1990, the only imaging techniques available for evaluating pulmonary circulation were ventilation-perfusion scintigraphy and pulmonary angiography. Kalender et al first reported the benefits of spiral CT in 1990 and since the the pioneering study by Remy-Jardin et al in 1992 there have been several large studies specifically evaluating the diagnostic accuracy of spiral computed tomographic pulmonary angiography (SCTPA). Spiral CT angiography is an accurate means of diagnosing pulmonary embolism with sensitivity and specifity in the region of $86 \%$ to $100 \%$ and $78 \%$ to $96 \%$ respectively ${ }^{49-51}$. Garg et al studied 44 patients with suspected pulmonary embolism and all underwent both V$\mathrm{P}$ scan and spiral CT. Compared both results with pulmonary angiography. They didn't only found the Spiral CT has greater accuracy and specificity than V-P scanning in patients but also found that $\mathrm{CT}$ provides alternative diagnosis in $31 \%$ of patient ${ }^{52}$. All the large studies that have evaluated the accuracy of CT have been restricted to the identification of emboli within the central, lobar and segmental arteries with sensitivity of $86 \% \%^{50,51,53}$. The prevalence of small sub-segmental emboli remains debatable with varying frequencies. Stein and colleagues ${ }^{53}$ found based on data from all patients with PE in PIOPED, the prevalence of PE limited to subsegmental pulmonary arteries is low, 6\%. A spiral CT angiography is currently unable to identify reliably emboli within pulmonary arteries below the fourth generation with sensitivity of only $30 \%{ }^{51,53}$. In a report by Remy Jarden and colleagues, visualization of subsegmental vessels were much better with a scanner equipped with 3-mm collimation than with 2-mm collimation ${ }^{54}$. Scanner equipped with 1-mm collimation increased the yield by $40 \%$ and also improved the inter-observer agreement ${ }^{55}$. Meta-analysis by Moores and colleagues, reviewed all published studies that used Computed tomography pulmonary angiography (CTPA) to evaluate suspected PE and reported at least 3 months of follow-up in patients not receiving anticoagulation on the basis of a negative CTPA result. The rate of subsequent venous thromboembolism after negative results on CTPA is similar to that seen after negative results on conventional pulmonary angiography. It appears to be safe to withhold anticoagulation after negative CTPA results ${ }^{56}$. In an another retrospective study by Nilsson and colleagues, only 4 patient out of 441 with initial negative CTPA and no DVT symptoms develop VTE during 3- month follow up ${ }^{57}$. A prospective study by Friera et al, out of the 99 patients, 9 (9\%) died during the 9-month follow-up, the cause of death in each case was not due to thromboembolic venous disease. No venous thromboembolic events were detected in the other 90 patients $^{58}$. Similar results were reported in other studies ${ }^{59-}$ ${ }^{63}$. The most reliable and basic criterion for the diagnosis of acute pulmonary embolism is the finding of an intraluminal filling defect. When contrast medium can flow around a central clot, the appearance is either of a "rail track" if the arteries lies parallel and within the scan plane or a "polo mint" if the artery runs perpendicular to the scan plane. Combined CT venography and pulmonary angiography was first tried in 1998 and since then this imaging methods is gaining popularity. PIOPED II study has been initiated to look at it's efficacy ${ }^{64}$. Preliminary results suggest the spiral $\mathrm{CT}$ and venous phase $\mathrm{CT}$ may becomes a first line diagnostic tools in evaluating pulmonary embolism. Advantages of combined CT venography and pulmonary angiography is that visualization of chest and extremities in one go, better accuracy in the evaluation of iliac veins, Inferior vena cava (IVC), and vessels poorly seen on sonography. CTVPA has now been studied by multiple researchers and showed very promising results and may become choice of procedures in future ${ }^{65-67}$.

\section{Magnetic Resonance Imaging (MRI)}

MRI has not been considered as a popular imaging method and the reason to put it down as signal loss due to cardiac pulsation and respiration, susceptibility artifacts caused by multiple air-tissue interfaces and low proton density. Recently, further improvements in MRI techniques have widened the potential for investigations of pulmonary disease. MRI is now being utilized to evaluate clinically suspected PE. The technique is rapid, accurate, avoids nephrotoxic iodinated contrast and is better accepted by patient than pulmonary angiography. There are more data available for diagnosing by spiral CT than by MRI at the present time but MRI has several attractive advantages, including excellent sensitivity and specificity for the diagnosing of DVT together with the potential for performing perfusion imaging. This technique may ultimate allow the simultaneous and accurate detection of both PE and DVT. Sostman et $\mathrm{al}^{68}$ reported in their study that the average sensitivity of CT was $75 \%$ and of MR $46 \%$; the average specificity of CT was $89 \%$ and of MR $90 \%$ and the conclusion was CT and MRI had similar accuracy for detecting pulmonary embolism. MRI can be useful in certain conditions namely pregnancy, pelvic vein thrombosis, pelvic malignancy, brachiocephalic and superior vena caval thrombosis. MRI is particularly helpful to recognize chronic $\mathrm{DVT}^{33,69}$. Lack of wide availability, difficult portability, high expenses and very few studies limits MRI as a first diagnostic tool for VTE at present. 


\section{Conclusion}

Until 1990, diagnosis of PE was a challenge and remained undiagnosed with noninvasive diagnostic tools. With the advances of technology and introduction of d-dimer testing, pulmonary embolism is diagnosed more frequently and negative test virtually rule out PE. CT pulmonary angiography is becoming the standard of care at many institutions for the evaluation of patients with suspected pulmonary embolism. But question raised about the effectiveness of spiral $\mathrm{CT}$ in the diagnosis of $\mathrm{PE}$ in critically ill patients ${ }^{70}$ and warrant further study in that particular population. There is increasing evidence that MRI could be useful tool in the diagnosis of PE, specially when intravenous contrast is contraindicated but more researches are needed. Increasing use of noninvasive methods as an additional means in pulmonary embolism diagnostics may in most cases spare the use of pulmonary angiography.

\section{Refernces}

1. Alikhan R, Peters F, Wilmott R, Cohen AT. Fatal pulmonary embolism in hospitalised patients: a necropsy review. J Clin Pathol. 2004 Dec;57(12):1254-7.

2. Goldhaber SZ, Morpurgo M. Diagnosis, treatment and prevention of pulmonary embolism, Report of the WHO/ISFC Task Force.JAMA 1992;268:1727-33.

3. Barritt DW, Jordan SC. Clinical features of pulmonary embolism. Lancet 1966;1: 729-32.

4. Goldhaber SZ, Visani L, De Rosa M. Acute pulmonary embolism: clinical outcomes in the International Cooperative Pulmonary Embolism Registry (ICOPER). Lancet 1999;353:1386-9.

5. Stein PD, Henry JW, Relyea B. Untreated patients with pulmonary embolism; outcome, clinical and laboratory assessment. Chest 1995; 107:931-35

6. Casazza F, Agostoni O, Mandelli V, Morpurgo M. The cardiologist facing pulmonary embolism. The experience of 160 cases of acute cor pulmonale Ital Heart J Suppl. 2000 Apr;1(4):520-6

7. Wells PS. Excluding Pulmonary Embolism at the Bedside without Diagnostic Imaging: Management of Patients with Suspected Pulmonary Embolism Presenting to the Emergency Department by Using a Simple. Clinical Model and d-dimer. Ann Intern Med. 2001 Jul 17;135(2):98-107.

8. Ginsberg WJS, Anderson DR, et al. Use of a clinical model for safe management of patients with suspected pulmonary embolism. Ann Intern Med 1998; 129 : 997-1005.

9. Petruzzelli PA, Donnamaria V, Giuntini C. The role of suspicion in the diagnosis of pulmonary embolism. Chest 1995;107:21S-24S.

10. PD Stein, SZ Goldhaber, JW Henry and AC Miller. Arterial blood gas analysis in the assessment of suspected acute pulmonary embolism.Chest 1996;109:78-81.

11. Meyer T, Binder L, Hruska N, Luthe H, Buchwald AB Cardiac troponin I elevation in acute pulmonary embolism is associated with right ventricular dysfunction. J Am Coll Cardiol. 2000 Nov 1;36(5):1632-6.

12. Mehta NJ, Jani K, Khan IA. Clinical usefulness and prognostic value of elevated cardiac troponin I levels in acute pulmonary embolism. Am Heart J 2003 May;145(5):821-5.

13. Yalamanchili K, Sukhija R, Aronow WS, Sinha N, Fleisher AG, Lehrman SG. Prevalence of increased cardiac troponin I levels in patients with and without acute pulmonary embolism and relation of increased cardiac troponin I levels with in-hospital mortality in patients with acute pulmonary embolism. Am J Cardiol 2004 Jan 15;93(2):2634.

14. Pruszczyk P, Kostrubiec M, Bochowicz A, et al. N-terminal probrain natriuretic peptide in patients with acute pulmonary embolism. Eur Respir J 2003 Oct; 22(4): 649-53.
15. Stein PD, Dalen JE, McIntyre KM, Sasahara AA, Wenger NK, Willis PW 3rd. The electrocardiogram in acute pulmonary embolism. Prog Cardiovasc Dis 1975 Jan-Feb;17(4):247-57.

16. Ferrari E, Imbert A, Chevalier T, Mihoubi A, Morand P, Baudouy $\mathrm{M}$. The ECG in pulmonary embolism. Predictive value of negative T waves in precordial leads--80 case reports. Chest .....; 111, 537-543.

17. Punukollu G, Gowda RM, Khan IA, Wilbur SL, Vasavada BC, Sacchi TJ. QT interval prolongation with global T-wave inversion: a novel ECG finding in acute pulmonary embolism. Ann Noninvasive Electrocardiol 2004 Jan;9(1):94-8.

18. Petrov DB. Appearance of right bundle branch block in electrocardiograms of patients with pulmonary embolism as a marker for obstruction of the main pulmonary trunk. J Electrocardiol 2001 Jul;34(3):185-8.

19. Akula R, Hasan SP, Alhassen M, Mujahid H, Amegashie E. Rightsided EKG in pulmonary embolism. J Natl Med Assoc 2003 Aug;95(8):714-7.

20. Tardy B, Tardy-Poncet B, Viallon A, Lafond P, Page Y, Venet C, Bertrand JC. Evaluation of D-dimer ELISA test in elderly patients with suspected pulmonary embolism. Thromb Haemost 1998 Jan;79(1):3841.

21. Flores J, Garcia-Avello A, Flores VM, Navarro JL, Canseco F, Perez-Rodriguez E. Tissue plasminogen activator plasma levels as a potential diagnostic aid in acute pulmonary embolism. Arch Pathol Lab Med 2003 Mar;127(3):310-5.

22. Kucher N, Kohler HP, Dornhofer T, Wallmann D, Lammle B. Accuracy of D-dimer/fibrinogen ratio to predict pulmonary embolism: a prospective diagnostic study. J Thromb Haemost. 2003 Apr;1(4):70813.

23. Stein PD, Terrin ML, Hales CA, Palevsky HI, Saltzman HA, Thompson BT, Weg JGClinical, laboratory, roentgenographic, and electrocardiographic findings in patients with acute pulmonary embolism and no pre-existing cardiac or pulmonary disease. Chest 1991 Sep;100(3):598-603.

24. Elliot C, Goldhaber S, Visani L, De Rosa M. Chest radiograph in acute pulmonary embolism. Results from the International Cooperative Pulmonary Embolism Registry. Chest 2000;118:33-38.

25. Miniati M, Prediletto R, Formichi B et al. Accuracy of clinical assessment in the diagnosis of pulmonary embolism.Am J Respir Crit Care Med. 1999; 159:864-71.

26. Kasper W, Geibel A, Tiede $\mathrm{N}$ et al. Distinguishing between acute and subacute massive pulmonary embolism by conventional and Doppler echocardiography. Br Heart J 1993;70:352-6.

27. McConnell MV, Solomon SD, Rayan ME, Come PC, Goldhaber SZ, Lee RT. Regional right ventricular dysfunction detected by echocardiography in acute pulmonary embolism. Am j cardiol. 1996;78:469-73.

28. Pruszczyk P, Torbicki A, Kuch-Wocial A, Pruszynski B, et al.Noninvasive diagnosis of suspected severe pulmonary embolism: transesophageal echocardiography vs spiral CT. Chest. 1997;112:722-8. 29. Ribeiro A, Juhlin-Dannfelt, Brodin LA, Hommgren A, Jorfeldt L. pulmonary embolism: relation between the degree of right ventricle overload and the extent of perfusion defects. Am. Heart J. 1998; 868-74. 30. Ribeiro A, Juhlin-Dannfelt, Brodin LA, Hommgren A, Jorfeldt L. Echocardiography Doppler in pulmonary embolism:right ventricular dysfunction as a predictor of mortality rate. Am Heart J. 1997;134:47987.

31. Eze AR, Comerota AJ, Kerr RP, Harada RN, Domeracki F. Is venous duplex imaging an appropriate initial screening test for patients with suspected pulmonary embolism? Ann Vasc Surg. 1996 May;10(3):220-3.

32. Peterson DA, Kazerooni EA, Wakefield TW, Knipp BS, Forauer AR, Bailey BJ, Sullivan VV, Proctor MC, Henke PK, Greenfield LJ, Stanley JC, Upchurch GR Jr Computed tomographic venography is specific but not sensitive for diagnosis of acute lower-extremity deep venous thrombosis in patients with suspected pulmonary embolus. J Vasc Surg. 2001 Nov;34(5):798-804.

33. Spritzer CE, Trotter P, Sostman HD: Deep vein thrombosis: Gradient-recalled -echo MR imaging changes over time.-experience in 10 patients. Radiology 1998;208:631-639.

34. Murphy TP, Cronan JJ: Evolution of Deep vein thrombosis: A prospective evaluation with US. Radiology 1990;177:543-48 
35. Killewich LA, Nunnelee JD, Auer AI. Value of lower extremity venous duplex examination in the diagnosis of pulmonary embolism. $\mathrm{J}$ Vasc Surg. 1993 May;17(5):934-8; discussion 938-9.

36. Daniel KR, Jackson RE, Kline JA. Utility of lower extremity venous ultrasound scanning in the diagnosis and exclusion of pulmonary embolism in outpatients. Ann Emerg Med. 2000 Jun;35(6):547-54.

37. Value of the ventilation/perfusion scan in acute pulmonary embolism. Results of the prospective investigation of pulmonary embolism diagnosis (PIOPED). The PIOPED Investigators. [PMID: 2332918] JAMA. 1990;263:2753-9.

38. Hagen P, Hartmann I, Hoekstra O, et al: Comparison of observer variability and accuracy of different criteria for lung scan interpretation. J. Nucl. Med. 2003;44:739-44.

39. Gotthardt M, Schipper M, Franzius C, Behe M, Barth A, Schurrat T, Hoffken H, Gratz S, Joseph K, Behr TM. Follow-up of perfusion defects in pulmonary perfusion scanning after pulmonary embolism: are we too careless? Nucl Med Commun. 2002 May;23(5):447-52.

40. Bauld DL, Kovacs MJ. Dalteparin in emergency patients to prevent admission prior to investigation for venous thromboembolism. [PMID: 9928688] Am J Emerg Med. 2000;83:416-20.

41. Schluger N, Henschke C, King T, Russo R, Binkert B, Rackson M, et al. Diagnosis of pulmonary embolism at a large teaching hospital. [PMID: 8083936] J Thorac Imaging. 1994;9:180-4.

42. Kember PG, Euinton HA, Morcos SK. Clinicians' interpretation of the indeterminate ventilation-perfusion scan report. [PMID: 9536900] $\mathrm{Br}$ J Radiol. 1997;70:1109-11.

43. Frankel N, Coleman RE, Pryor DB, Sostman HD, Ravin CE. Utilization of lung scans by clinicians. [PMID: 3712055] J Nucl Med. 1986;27:366-9.

44. Scott, James A. The lung scan and the abnormal chest X-ray: difficult diagnoses. Nuclear Medicine Communications. 25(11):11371141, November 2004.

45. Stein PD, Coleman RE, Gottschalk A, Saltzman HA, Terrin ML Weg JG. Diagnostic utility of ventilation/perfusion lung scans in acute pulmonary embolism is not diminished by pre-existing cardiac or pulmonary disease. Chest. $1991 \mathrm{Sep} ; 100(3): 604-6$.

46. Kahn D, Bushnell DL, Dean R, Perlman SB. Clinical outcome of patients with a 'low probability' of pulmonary embolism on ventilationperfusion lung scan. Arch Intern Med. 1989 Feb;149(2):377-9.

47. Miniati M, Pistolesi M, Marini C, Di Ricco G, Formichi B, Prediletto R, Allescia G, Tonelli L, Sostman HD, Giuntini C. Value of perfusion lung scan in the diagnosis of pulmonary embolism: results of the Prospective Investigative Study of Acute Pulmonary Embolism Diagnosis (PISA-PED). Am J Respir Crit Care Med. 1996 Nov;154(5):1387-93.

48. Reinartz P, Wildberger JE, Schaefer W, Nowak B, Mahnken AH Tomo graphic imaging in the diagnosis of pulmonary embolism: a comparison between V/Q lung scintigraphy in SPECT technique and multislice spiral CT. J. Nucl. Med. 2004 Sep;45(9):1501-8.

49. Perrier A, Howarth N, Didier D, et al: Performance of Helical computed tomography in unselected patients with suspected pulmonary embolism. Ann Intern Med. 2001;135:88-97.

50. Kim K, Muller N, Mayo J,: Clinically suspected pulmonary embolism:utility of spiral CT.Radiology 1999;210:693-697.

51. van Strijen M, de Monye W, kieft GJ, et al: Diagnosis of pulmonary embolism with spiral CT: A prospective cohort study in 617 patients. Radiology 1999(suppl);213:127.

52. Garg K, Welsh CH, Feyerabend AJ, Subber SW, Russ PD, Johnston RJ, Durham JD, Lynch DA. Pulmonary embolism: diagnosis with spiral $\mathrm{CT}$ and ventilation-perfusion scanning--correlation with pulmonary angiographic results or clinical outcome. Radiology. 1998 Jul;208(1):201-8.
53. Stein P, Henry J: Prevalence of acute pulmonary embolism in central and subsegmental pulmonary arteries and relation to probability interpretation of ventilation/perfusion scans. Chest 1997;11:1246-48.

54. Remy-Jardin M, Remy J, Artaud D. et al: Peripheral pulmonary arteries: Optimization of the spiral CT acquisition protocol. Radiology 1997;204:157-163.

55. Schoepf U, Holzknecht N, Helmberger T, et al: Subsegmental pulmonary emboli: Improved detection with thin collimation multi detector row spiral CT. Radiology 2002; 222:483-90.

56. Moores LK, Jackson WL Jr, Shorr AF, Jackson JL Meta-analysis: outcomes in patients with suspected pulmonary embolism managed with computed tomographic pulmonary angiographyAnn Intern Med. 2004 Dec 7;141(11):866-74.

57. Nilsson T, Olausson A, Johnsson H, Nyman U, Aspelin P. Negative spiral CT in acute pulmonary embolismActa Radiol. 2002 Sep;43(5):486-91.

58. Friera A, Olivera MJ, Suarez C, Gimenez N, Caballero P;Thromboembolic Study Group of La Princesa Hospital. Clinical validity of negative helical computed tomography for clinical suspicion of pulmonary embolism. Respiration. 2004 Jan-Feb;71(1):30-6.

59. Donato AA, Scheirer JJ, Atwell MS, Gramp J, Duszak R Jr. Clinical outcomes in patients with suspected acute pulmonary embolism and negative helical computed tomographic results in whom anticoagulation was withheld. Arch Intern Med. 2003 Sep 22;163(17):2033-8.

60. Lomis NN, Yoon HC, Moran AG, Miller FJ. Clinical outcomes of patients after a negative spiral CT pulmonary arteriogram in the evaluation of acute pulmonary embolism. J Vasc Interv Radiol. 1999 Jun;10(6):707-12.

61. Swensen SJ, Sheedy PF 2nd, Ryu JH, Pickett DD, Schleck CD, Ilstrup DM, Heit JAOutcomes after withholding anticoagulation from patients with suspected acute pulmonary embolism and negative computed tomographic findings: a cohort study. Mayo Clin Proc. 2002 Feb;77(2):130-8.

62. Tillie-Leblond I, Mastora I, Radenne F, Paillard S, Tonnel AB, Remy J, Remy-Jardin M. Risk of pulmonary embolism after a negative spiral CT angiogram in patients with pulmonary disease: 1-year clinical follow-up study. Radiology. 2002 May;223(2):461-7.

63. Krestan CR, Klein N, Fleischmann D, Kaneider A, Novotny C, Kreuzer S, Riedl C, Minar E, Janata K, Herold CJ. Value of negative spiral CT angiography in patients with suspected acute PE: analysis of PE occurrence and outcome. Eur Radiol. 2004 Jan;14(1):93-8.

64. Gottsschalk A, Stein P, Goodman L R, and Sostman H D. Overview of the prospective investigation of pulmonary embolism diagnosis II. Semin Nucl. Med 2002;32:1173-182

65. Loud P, Katz D, Bruce D: Deep vein thrombosis with suspected pulmonary embolism. Detection with combined CT venography and pulmonary angiography. Radiology 2001;219:498-502.

66. Bruce D, Loud P, Klippenstein et al: Combined CT venography and pulmonary angiography. How much venous enhancement is routinely obtained? AJR AM J Roentgenol 2001;176:1281-1285.

67. Bourriot $\mathrm{K}$, Couffinhal $\mathrm{T}$ et al. Clinical outcome after a negative spiral CT pulmonary angiographic finding in an inpatient population from cardiology and pneumology wards.Chest.2003;123(2):359-65.

68. Sostman HD, Layish DT, Tapson VF, Spritzer CE, DeLong DM, Trotter P, MacFall JR, Patz EF Jr, Goodman PC, Woodard PK, Foo TK, Farber JL. Prospective comparison of helical CT and MR imaging in clinically suspected acute pulmonary embolism. J Magn Reson Imaging. 1996 Mar-Apr;6(2):275-81.

69. Polak JF, Fox LA: MR assessment of the extremity veins. Semin US CT MR. 1999;20:36-46.

70. Velmahos G C, Toutouzas $\mathrm{K}$ G et al. Can we rely on computed scanning to diagnose pulmonary embolism in critically ill surgical patients? J. trauma. 2004;56(3):518-25. 